\title{
Effects of Non-covalent Interaction of Dimethyl Suberimidate on Lipase Stability
}

\author{
Masaya Kawase, Kenji Sonomoto and Atsuo TanaKa \\ Laboratory of Industrial Biochemistry, Department of Industrial Chemistry, \\ Faculty of Engineering, Kyoto University, Yoshida, \\ Sakyo-ku, Kyoto 606, Japan \\ Received April 5, 1990
}

\begin{abstract}
The hydrolytic activity of the purified yeast lipase OF 360 was greatly decreased by heating above $45^{\circ} \mathrm{C}$ and the residual activity after heating at $50^{\circ} \mathrm{C}$ for $15 \mathrm{~min}$ was about one-tenth of the original. Treatment of the lipase with a bifunctional reagent, dimethyl suberimidate (DMS), increased the heat stability. Stabilization by DMS treatment was supposed to be caused by non-covalent interaction between the lipase and DMS through electrostatic and/or hydrophobic forces. In addition, analysis of FT-IR spectra of the DMS- and non-treated lipases suggested that the main skeleton of the treated lipase was little perturbed by heating.
\end{abstract}

The unique catalytic properties of enzymes have been widely used in synthetic organic chemistry and chemical industries. However, the negative aspects inherent in biocatalysts, such as instability toward denaturation by heat, restrict their much wider use. Therefore, many studies have been done to improve the functions and properties of enzymes by chemical and biological methods. Covalent interaction by chemical modification is one of the most hopeful approaches to preparing stable enzymes. ${ }^{1)}$ Unfortunately, modification of amino acid residues of enzymes at a specific position is scarcely possible without several functions of enzymes themselves being lowered.

Several additives, such as glycerol and dithiothreitol, can stabilize enzymes. However, such an effect of additives disappears rapidly upon dialysis of the enzyme solution and so on because these additives interact with enzymes by weak intermolecular forces in solution. It is essential for enzyme stability to keep a certain concentration of the additives in the vicinity of the enzymes. This is uneconomical and troublesome for the use of enzymes on an industrial scale. If such a non-covalent interaction is allowed to become stronger in practical uses, we can develop a new field of chemical modification for stabilization of enzymes with desired functions in addition to conventional covalent interaction methods.

Lipase (triacylglycerol acylhydrolase, EC 3.1.1.3) is known to catalyze the hydrolysis, ${ }^{2}$ synthesis, ${ }^{3,43}$ and group exchange ${ }^{5)}$ of esters, and is used for diverse purposes. We have studied the improvement of functions of lipase OF 360 from Candida cylindracea by chemical modification. ${ }^{6,7)}$ During these studies, it was found that the heat stability of the lipase was increased somewhat by the addition of dimethyl suberimidate (DMS). Furthermore, this stabilization with DMS was kept even after dialysis of the treated lipase. Exploration of this effect of DMS on the lipase stability may give basic information for developing new and simple stabilizing methods.

In this paper, we report the optimization of DMS treatment and clarify this stabilizing effect by characterization of the treated lipase.

\section{Materials and Methods}

Enzyme. A crude lipase preparation (lipase OF 360) from Candida cylindracea was purchased from Meito Sangyo Co., Tokyo, Japan. Lipase was purified by the method 
reported previously. ${ }^{6)}$

Analytical methods. Protein was assayed by the method of Lowry et al. ${ }^{8)}$ in which bovine serum albumin was used as the standard. Hydrolytic activity of lipase was measured at $30^{\circ} \mathrm{C}$ by the method of Kurooka et al. ${ }^{9)}$ using 2,3-dimercaptopropan-1-ol tributyrate as a substrate.

Chemical treatment of lipase OF 360 . Purified lipase OF 360 was treated with dimethyl suberimidate (DMS). Unless stated otherwise, the treatment was done under the following conditions. The lipase $(6 \mu \mathrm{M})$ was treated with $10 \mathrm{~mm}$ DMS at $30^{\circ} \mathrm{C}$ in $50 \mathrm{~mm}$ phosphate buffer ( $\mathrm{pH} \mathrm{7.65)}$ for $1 \mathrm{hr}$. The reaction was started by adding a freshly prepared solution of a modifying reagent to the enzyme solution. After the chemical treatment described above, excess reagent was removed by dialysis against $50 \mathrm{~mm}$ phosphate buffer $(\mathrm{pH} 7.20)$ at $20^{\circ} \mathrm{C}$ overnight.

Measurement of heat stability. Unless stated otherwise, lipase $(6 \mu \mathrm{M})$ was heated at $50^{\circ} \mathrm{C}$ for $15 \mathrm{~min}$ and then cooled rapidly in ice-water. Thereafter, lipase was incubated at $20^{\circ} \mathrm{C}$ for $15 \mathrm{~min}$, and then the activity was measured as mentioned above.

Analysis of the treated lipase. The number of free amino groups of the DMS-treated lipase was measured by a modification of Habeeb's method ${ }^{10)}$ as follows. After the treated lipase $(1.5 \mu \mathrm{M})$ was mixed with $1.4 \mathrm{~mm}$ trinitrobenzenesulfonic acid, $0.25 \mathrm{~mm}$ sodium tetraborate, and

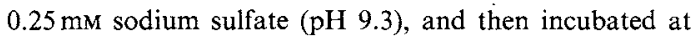
$40^{\circ} \mathrm{C}$ for $20 \mathrm{~min}$, free amino groups of the lipase were measured from the absorbance at $410 \mathrm{~nm}$. Ammonium hydroxide was used as a standard. The structure of the treated lipase was analyzed by a Shimadzu FT-IR 4100 spectrometer with a refraction method.

Chemicals. DMS was obtained from Nacalai Tesque, Inc., Kyoto, Japan, and hexamethylenediamine from Wako Pure Chemical Industries, Ltd., Osaka, Japan. Other chemicals were also purchased from commercial sources.

\section{Results and Discussion}

Lipase OF 360 lost its hydrolytic activity rapidly upon being heated above $45^{\circ} \mathrm{C}$. The heat stability was, therefore, evaluated by the residual activity of the lipase after heating at $50^{\circ} \mathrm{C}$ for $15 \mathrm{~min}$ to optimize the conditions of treatment with dimethyl suberimidate (DMS). From the results concerning treatment $\mathrm{pH}$ (7.10-8.70), kind of buffers (HEPES, phosphate, and borate), DMS concentration (up to $100 \mathrm{~mm})$, treatment time $(0.5-2 \mathrm{hr})$, and treat- ment temperature $\left(040^{\circ} \mathrm{C}\right)$, the optimized DMS treatment conditions were decided as follows; lipase OF $3606 \mu \mathrm{M}$, DMS $10 \mathrm{mM}$, temperature $30^{\circ} \mathrm{C}$, treatment time $1 \mathrm{hr}$, and $50 \mathrm{~mm}$ phosphate buffer ( $\mathrm{pH} 7.65$ ). Table I shows the heat stability of DMS- and non-treated lipase OF 360. Although the residual activity of the non-treated enzyme was only about one-tenth after heating at $50^{\circ} \mathrm{C}$ for $15 \mathrm{~min}$, the DMS-treated lipase dialyzed as mentioned in Materials and Methods kept about $30 \%$ of its original activity, indicating clearly the effectiveness of the DMS treatment. However, the stability of lipase OF 360 at $60^{\circ} \mathrm{C}$ was not improved by this treatment. DMS treatment also activated the lipase slightly when heated at 30 or $40^{\circ} \mathrm{C}$ for $15 \mathrm{~min}$.

To clarify this effect of DMS treatment, we attempted to characterize the treated lipase. As shown in Fig. 1, the molecular mass of the lipase did not change even after DMS treatment. This result suggested that lipase OF 360 was not crosslinked intermolecularly with DMS under these conditions. To find out whether DMS reacts or not with the lipase covalently, the number of free amino groups in the lipase protein was measured by the modified trinitrobenzenesulfonic acid method. ${ }^{10)}$ Excess DMS molecules were removed by dialysis after the chemical treatment of the lipase as described in Materials and Methods. As shown in Table II, DMS treatment at $30^{\circ} \mathrm{C}$,

Table I. Heat STability OF DMS-Treated LIPASE OF 360

\begin{tabular}{ccc}
\hline \multirow{2}{*}{$\begin{array}{c}\text { Temperature } \\
\text { for heating }\left({ }^{\circ} \mathrm{C}\right)\end{array}$} & \multicolumn{2}{c}{ Residual activity ${ }^{a}$ (units) } \\
\cline { 2 - 3 } & $\begin{array}{c}\text { Non-treated } \\
\text { lipase }\end{array}$ & $\begin{array}{c}\text { DMS-treated } \\
\text { lipase }\end{array}$ \\
\hline 20 & $7280(100 \%)$ & $6620(100 \%)$ \\
30 & $7350(101)$ & $8410(127)$ \\
35 & $6700(92)$ & $7680(116)$ \\
40 & $7500(103)$ & $8610(130)$ \\
45 & $5820(80)$ & $6490(98)$ \\
50 & $950(13)$ & $2180(33)$ \\
60 & $40(1)$ & $40(1)$ \\
\hline
\end{tabular}

a Residual activity was measured at $30^{\circ} \mathrm{C}$ after heating at the indicated temperature for $15 \mathrm{~min}$. 
which improved the lipase stability, increased the free amino groups, but the number of free amino groups was decreased by DMS treatment at $0^{\circ} \mathrm{C}$, which provided no enhancement of heat stability. These results indicated that covalent bonds between amino groups of the lipase and DMS were formed during DMS treatment at $0^{\circ} \mathrm{C}$ but not at $30^{\circ} \mathrm{C}$. Thus, the effect of DMS on stabilization of the lipase did not result from intramolecular or inter-
$94.0 \mathrm{kD}-$

$67.0 \mathrm{kD}-$

43.0 kD -

\section{$20.1 \mathrm{kD}-$}
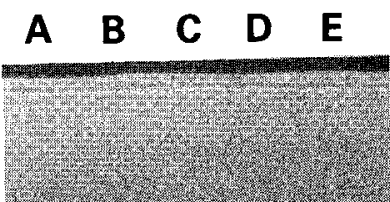

molecular crosslinking but might be derived from the interaction of DMS with the lipase via electrostatic and/or hydrophobic forces. The increase in the number of free amino groups by DMS treatment at $30^{\circ} \mathrm{C}$ would be caused by the artificial chemical conversion of DMS itself conjugated with the lipase into the corresponding diamino-substituted compound under alkaline conditions ( $\mathrm{pH}$ 9.3) of the amino group measurement as shown in Fig. 2. The following experiment was done to calculate the number of DMS conjugated with the lipase. The number of free amino groups was calculated in DMS (1 mM) in $50 \mathrm{~mm}$ phosphate buffer ( $\mathrm{pH}$ 7.2) without the lipase by a modification of Habeeb's method as described in Materials and Methods. One $\mu \mathrm{mol}$ of DMS gave $0.105 \mu \mathrm{mol}$ of the diamino-substituted compound according to the reaction illustrated

Table II. Number of FreE Amino Groups IN LIPASE OF 360

\begin{tabular}{cc}
\hline Lipase OF 360 & $\begin{array}{c}\text { Number of free } \\
\text { amino groups }\end{array}$ \\
\hline $\begin{array}{c}\text { Non-treated } \\
\text { DMS-treated }\end{array}$ & 16.6 \\
at $0^{\circ} \mathrm{C}^{b}$ & 13.9 \\
at $30^{\circ} \mathrm{C}^{b}$ & 20.5
\end{tabular}

The results are the mean value of 6 experiments and the standard errors are within $10 \%$ of the mean value.

a Expressed as number per molecule of the lipase.

$b$ DMS treatment of lipase was done at the indicated temperature. The other conditions are described in Materials and Methods.
Fig. 1. SDS-Polyacrylamide Gel Electrophoresis of DMS-treated Lipase OF 360.

Lipase OF 360 was treated at an indicated DMS concentration under the conditions described in Materials and Methods. A, DMS 0 mM; B, DMS $0.1 \mathrm{~mm}$; C, DMS $1 \mathrm{~mm}$; D, DMS $10 \mathrm{~mm}$; E, DMS $100 \mathrm{~mm}$.
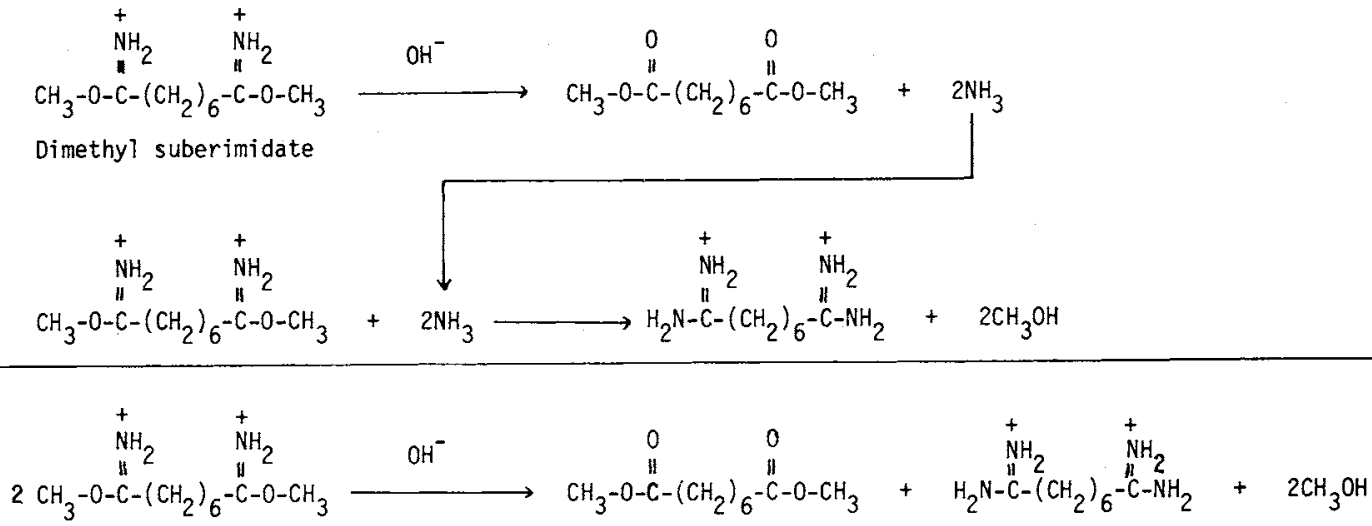

Fig. 2. Chemical Conversion of DMS into the Diamino-substituted Compound under Alkaline Condition. 
in Fig. 2. By using the conversion ratio obtained, the number of DMS conjugated with the lipase can be calculated from the results in Table II. It was found that nineteen molecules of DMS might be conjugated with one molecule of the lipase.

Furthermore, we attempted to confirm the non-covalent interaction of DMS with the lipase via electrostatic and/or hydrophobic forces. The DMS-treated lipase was incubated with $20 \%$ ethanol in $50 \mathrm{~mm}$ phosphate buffer (pH 7.2) at $30^{\circ} \mathrm{C}$ for $1 \mathrm{hr}$, and then dialyzed. The lipase obtained showed the same heat stability as the native lipase, of which the number of free amino groups was equal to the native one. These results suggested the existence of the lipase-DMS complex formed by non-covalent interaction described above.

When lipase OF 360 was treated with $10 \mathrm{~mm}$ hexamethylenediamine instead of DMS under the optimized conditions described above, the heat stability of the treated lipase was almost the same as that of the non-treated enzyme. This suggested that improvement of lipase stability by DMS treatment might not be caused only by hydrophobic interaction because hexamethylenediamine has the same main skeleton as DMS. The electrostatic force of the imidate groups of DMS would be essential not only for conjugation of DMS with the lipase but also for stabilization of the enzyme. Dimethyl adipimidate (DMA), with tetramethylene as a main chain, also provided a moderate enhancement of heat stability of the lipase. Chain length of the modifiers is considered to be one of the important factors for preparation of a heat-stable lipase.

The structure of the main skeleton of the
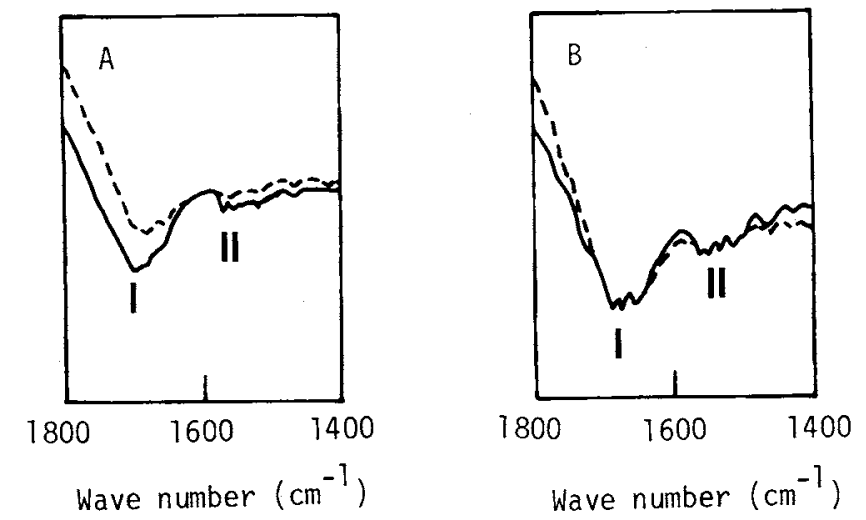

Wave number $\left(\mathrm{cm}^{-1}\right)$
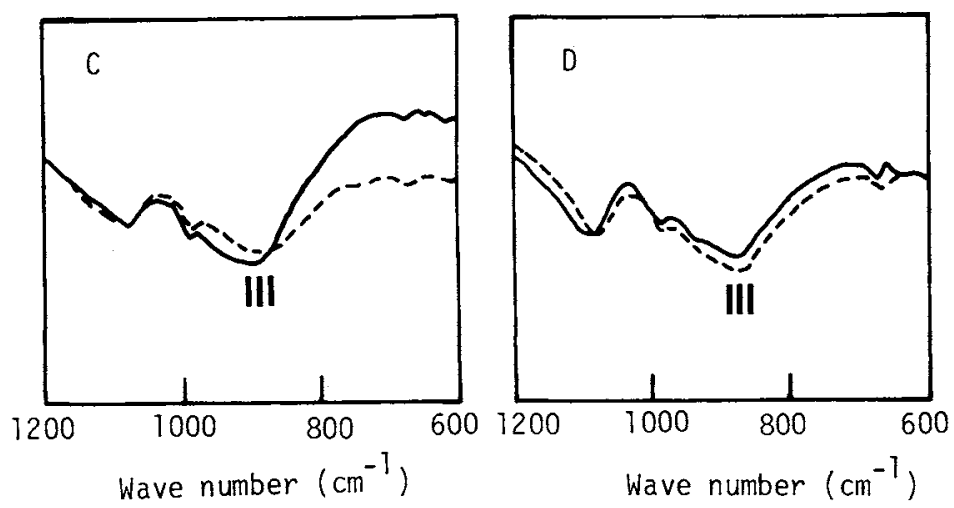

Fig. 3. FT-IR Spectra of Lipase OF 360 .

A, C, non-treated lipase; B, D, DMS-treated lipase; - , before heating; ---, after heating at $50^{\circ} \mathrm{C}$ for $15 \mathrm{~min}$. The details in peaks I, II and III are described in the text. 
DMS-treated lipase was studied with Fourier transform infrared spectroscopy (FT-IR) (Fig. 3 ), as this method has been recently used for the conformational study of proteins. ${ }^{1-13)}$ No difference between the DMS- and non-treated lipases was observed at the peaks $\mathrm{I}(\mathrm{C}=\mathrm{O}$ stretching; amide I) and II (N-H stretching; amide $\mathrm{II})^{14)}$ (Figs. 3A and $\mathrm{B}$ ), indicating that the DMS treatment caused no significant change to the structure of the lipase. On the other hand, a small difference was observed at peak III (overlap of various kinds of vibration of amino acid residues), ${ }^{14}$ that is, peak III was at $900 \mathrm{~cm}^{-1}$ with the non-treated lipase, but at $877 \mathrm{~cm}^{-1}$ with the DMS-treated enzyme (Figs. $3 \mathrm{C}$ and $\mathrm{D}$ ). As peak III gives information about the state of intramolecular interaction between the amino acid residues, the difference observed at peak III might be caused by the conjugation of DMS with the lipase.

The positions of peaks I-III in the spectrum of the DMS-treated lipase were scarcely changed even by heating at $50^{\circ} \mathrm{C}$ for $15 \mathrm{~min}$, while peaks I and III shifted to the lower wave number side by 17 and $24 \mathrm{~cm}^{-1}$, respectively, in the case of the non-treated lipase. The deviation of peak $\mathrm{I}$ is due to the loosened folding of the lipase by heating, because the position of the peak reflects the tightness of the folding of a protein and a hypsocromic shift is observed with the loosening of the structure. The deviation of peak III is also explained by local change of the protein structure. Thus, FT-IR analysis indicates that the non-treated lipase is liable to be unfolded by heating compared to the DMS-treated enzyme, the main skeleton of which is little perturbed by heating owing to the complexing of the lipase with DMS, supporting the heat resistance of the DMS-treated lipase.

As mentioned above, the yeast lipase was moderately stabilized by non-covalent interaction with DMS. This effect of DMS was maintained even after dialysis of the treated lipase. Non-covalent interaction on enzyme stability shown here gives a useful and newly important guide to the field of improvement of enzyme functions by chemical modification and treatment methods. Integration of immobilization methods and this simple treatment method will also create new potent biocatalysts to be used in biotechnology.

Acknowledgment. We thank to Prof. Zen-ichiro Takehara, Dr. Zempachi Ogumi, and Mr. Yoshiharu Uchimoto, Department of Industrial Chemistry, Faculty of Engineering, Kyoto University, for the measurement of FT-IR spectra.

\section{References}

1) V. P. Torchilin and K. Martinek, Enzyme Microb. Technol., 1, 74 (1979).

2) Y. Kimura, A. Tanaka, K. Sonomoto, T. Nihira and S. Fukui, Eur. J. Appl. Microbiol. Biotechnol., 17, 107 (1983).

3) T. Kawamoto, K. Sonomoto and A. Tanaka, Biocatalysis, 1, 137 (1987).

4) S. Koshiro, K. Sonomoto, A. Tanaka and S. Fukui, J. Biotechnol, 2, 47 (1985).

5) K. Yokozeki, S. Yamanaka, K. Takinami, Y. Hirose, A. Tanaka, K. Sonomoto and S. Fukui, Eur. J. Appl. Microbiol. Biotechnol., 14, 1 (1982).

6) M. Kawase and A. Tanaka, Enzyme Microb. Technol., 11, 44 (1989).

7) M. Kawase and A. Tanaka, Biotechnol. Lett., 10, 393 (1988).

8) O. H. Lowry, N. J. Rosebrough, A. L. Farr and R. J. Randall, J. Biol. Chem., 193, 265 (1951).

9) S. Kurooka, S. Okamoto and M. Hashimoto, $J$. Biochem., 81, 361 (1977).

10) A. F. S. A. Habeeb, Anal. Biochem., 14,328(1966).

11) D. M. Byler and H. J. Susi, J. Indust. Microbiol., 3, 73 (1988).

12) K. J. Payne and A. Veis, Biopolymers, 27, 1749 (1988).

13) G. R. Sauer and R. E. Wuthier, J. Biol. Chem., 263, 13718 (1988).

14) R. M. Silverstein, G. C. Bassler and T. C. Morrill, "Spectromeric Identification of Organic Compounds," 4th Ed., John Wiley \& Sons, Inc., New York, 1981. 